\title{
Low molecular weight heparin in COVID-19 patients prevents delirium and shortens hospitalization
}

\author{
Damiano D'Ardes ${ }^{1}$ (D) Claudia Carrarini ${ }^{2}$ (D) $\cdot$ Mirella Russo $^{2}$ (D) $\cdot$ Fedele Dono $^{2}$ (D) $\cdot$ Rino Speranza $^{2} \cdot$ Anna Digiovanni $^{2}$. \\ Giovanni Martinotti ${ }^{2}$ (D) Angelo Di lorio $^{1}$ (D) $\cdot$ Marco Onofrj $^{2}$ (D) $\cdot$ Francesco Cipollone $^{1}$ (D) $\cdot$ Laura Bonanni $^{2}$ (D)
}

Received: 9 August 2020 / Accepted: 5 November 2020 / Published online: 13 November 2020

(C) Fondazione Società Italiana di Neurologia 2020

\begin{abstract}
Background COVID-19 patients present with delirium during their hospitalization.

Aims To assess the incidence of delirium in hospitalized COVID-19 patients and analyze the possible association with demographic, clinical, laboratory, and pharmacological factors.

Methods COVID-19 patients were assessed for clinical signs of delirium and administered the assessment test for delirium and cognitive impairment (4AT) and the Confusion Assessment Method for the Intensive Care Unit (CAM-ICU) scales.

Results Out of the 56 patients of our cohort, $14(25.0 \%)$ experienced delirium. The use of low molecular weight heparin (LMWH) (enoxaparin $1 \mathrm{mg} / \mathrm{kg} /$ daily) was less frequent in patients with delirium $(p=0.004)$ and was accompanied by lower $\mathrm{C}$ reactive protein $(\mathrm{CRP})$ levels $(p=0.006)$.

Discussion The use of LMWH was associated with absence of delirium, independently of comorbidities and age.

Conclusions The use of LMWH may help preventing the occurrence of delirium in COVID-19 patients, with possible reduction of length of stay in the hospital and sequelae.
\end{abstract}

Keywords Delirium $\cdot$ COVID-19 $\cdot$ Low molecular weight heparin $\cdot \mathrm{C}$ reactive protein

\section{Introduction}

Coronavirus disease (COVID-19) represents an enormous new threat to healthcare systems. As the infection has reached the pandemic stage, it has become clear that the older population is most vulnerable to severe respiratory infection with increased mortality [1]. Delirium is a well-recognized complication of respiratory illness in older adults [2].

Delirium in hospitalized patients is a strong independent predictor of mortality, increased hospital length of stay (LOS), cognitive impairment in the long term, increased cost of care, and subsequent hospitalizations [3].

Early studies indicate that 20-30\% of COVID-19 patients will present with or develop delirium during their hospitalization, with rates of 60-70\% in cases of severe illness [4].

Laura Bonanni

1.bonanni@unich.it

1 Department of Medicine and Aging Sciences, University G. d'Annunzio of Chieti-Pescara, Chieti, Italy

2 Department of Neuroscience, Imaging and Clinical Sciences, University G. d'Annunzio of Chieti-Pescara, Chieti, Italy
Hospital management of COVID-19 involves precautional isolation and contact with hospital staff mediated by personal protective equipment, which can be depersonalizing and frightening to the elderly, particularly those with underlying cognitive impairment. Thus, the current management approach may exacerbate and prolong the duration of delirium, with consequent poor outcomes and accelerated mortality.

In the present study, we assessed the incidence of delirium in COVID-19 patients hospitalized in an internal medicine unit and analyzed the possible association of delirium with demographic, clinical factors and pharmacological interventions.

\section{Methods}

We followed COVID-19 patients with moderate symptoms (positive swab for SARS-CoV-2 in patients with fever and/ or cough associated to CT scan with signs of interstitial pneumonia and/or to respiratory failure without need of mechanical ventilation) admitted between April the 1st and the 15th 2020 to the COVID-19 Unit of SS. Annunziata Hospital, Chieti, Italy, and followed until complete healing (remission of 
clinical signs and two serial negative swab tests). The patients were assessed for clinical signs of delirium and administered the assessment test for delirium \& cognitive impairment (4AT) and the Confusion Assessment Method for the Intensive Care Unit (CAM-ICU) scales.

Laboratory parameters (including blood count, creatinine, transaminases, electrolytes, urine test, arterial blood gas analysis, and C-reactive protein, CRP), comorbidities (hypertension, diabetes, cardiac failure, active cancer, arrhythmias, chronic obstructive pulmonary disease), other neurological symptoms (and neuroradiological features in 12 patients, supplementary Table 1), and pharmacological interventions were also recorded. A regional review board has approved the use of humans for this study.

\section{Statistical analysis}

Data were reported as mean \pm standard error (se) for continuous variables and as absolute number and percentage for categorical and ordinal variables. Differences between patients with and without delirium were evaluated with general linear model for continuous variables, and with Chi-square test for categorical or ordinal variables, Fischer's correction was applied if necessary.

To assess the independent effect of different variables in predicting the risk of delirium, logistic regression analysis was applied. Variables were considered in the models as dummy except for age that was modeled as continuous.
All the variables reaching a $p$ value $\leq 0.05$ at the univariate analysis were included in the multivariate logistic regression analysis predicting the risk of delirium. In details, low molecular weight heparin (LMWH), age, and comorbidities were considered in the model as dummy variables; the presence of less than two comorbidities was the reference group.

Mixed linear models were applied to assess differences between time of the study and presence of delirium in laboratory parameters. All analyses were performed with SAS 9.4.

\section{Results}

Fifty-six patients were recruited. Fourteen $(25.0 \%)$ experienced delirium during the hospitalization with $4 \mathrm{AT}$ and CAM scores higher than those found in patients not experiencing delirium $(p<0.001)$.

Patients with delirium were older than patients without signs of delirium $(p=0.002)$.

Two or more comorbidities were reported in $85 \%$ of patients who experienced delirium and in $40 \%$ of patients without delirium $(p=0.003)$. Table 1 summarizes the characteristics of the patients' populations.

CRP resulted to be higher and stable overtime in patients with delirium as compared with patients who did not experience delirium ( $p=0.006$ ) (Table 2).

The use of LMWH (enoxaparin administered at $1 \mathrm{mg} / \mathrm{kg} /$ daily) was less frequent in patients who developed delirium
Table 1 Differences between subjects who experienced delirium compared with patients without delirium

\begin{tabular}{llll}
\hline & Patients with delirium & Patients without delirium & $p$ value \\
\hline Age (years) & 14 & 42 & 0.002 \\
Sex male & $84.29 \pm 7.45$ & $70.98 \pm 14.86$ & 0.73 \\
LMWH & $5(38.5)$ & $14(33.3)$ & 0.004 \\
Antibiotics & $4(28.6)$ & $30(71.4)$ & 0.56 \\
Hydroxychloroquine & $0(0.0)$ & $4(9.5)$ & 0.70 \\
Other drugs & $3(21.4)$ & $7(16.7)$ & 0.10 \\
Neurological signs & $2(14.3)$ & $16(38.1)$ & 0.85 \\
Myalgia & $3(21.4)$ & $10(23.8)$ & 0.56 \\
Neuropathy & $0(0.0)$ & $4(9.5)$ & 0.57 \\
Vertigo & $0(0.0)$ & $3(7.1)$ & 0.44 \\
Comorbidities & $1(7.1)$ & $1(2.4)$ & 0.02 \\
Comorbidities $(\geq 2)$ & $2.93 \pm 1.40$ & $1.69 \pm 1.66$ & 0.003 \\
4AT & $12(85.7)$ & $17(40.5)$ & $<0.001$ \\
CAM-ICU & $8.21 \pm 3.66$ & $0.79 \pm 1.68$ & $<0.001$ \\
\hline
\end{tabular}

Data were reported as mean $\pm \mathrm{SE}$ for continuous variables and as absolute number (percentage) for categorical and ordinal variables. Significant differences are highlighted in italicized character

$L M W H$ low molecular weight heparin, $4 A T$ assessment test for delirium and cognitive impairment, $C A M-I C U$ Confusion Assessment Method for the Intensive Care Unit 
than in patients without signs of delirium $(p=0.004)$. Ten out of 14 patients with delirium and 12 out of 42 who did not experience delirium were not treated with LMWH due to contraindications reported in supplementary Table 2.

The prescription of LMWH seemed to have a protective effect from delirium (OR: 0.08 ; 95\% CI: $0.1-0.50 ; p=0.007$ ), independently of comorbidities (OR: 17.82 ; 95\% CI: $2.07-$ 153.08; $p=0.009$ ) and age.

In the logistic model sub-analysis, where only those subjects with a higher comorbidity score ( $\geq 2$ diseases) were analyzed, the results did not change; LMWH-prescription was the only statistically significant predictor of prevention of delirium.

LOS was longer in patients untreated with LMWH $(27.0 \pm$ 14.7 vs. $21.5 \pm 10.1 ; p=0.04$ ) independently of age and CAM score.

\section{Discussion}

Fourteen out of 56 patients (25\%) admitted to our COVID-19 Unit experienced delirium.

They were older than patients without signs of delirium and presented with a higher number of comorbidities, as expect$\mathrm{ed}^{4}$. No difference was found in the prevalence of other neurological symptoms associated with COVID- 19 .

We found a strong association between the use of LMWH and absence of delirium, independently of comorbidities and age.

Heparin has been implicated in binding to COVID-19 spike proteins as well as down-regulating interleukin-6 (IL6) [5], which has been shown to be elevated in COVID-19 patients [6], and thus, LMWH remains as the best choice of anticoagulant for admitted patients.

Table 2 Mixed linear models. Differences in laboratory parameters between times of the study according to delirium manifestation, during the hospitalization

\begin{tabular}{|c|c|c|c|c|c|c|c|}
\hline & \multicolumn{2}{|c|}{ Patients without delirium } & \multicolumn{2}{|c|}{ Patients with delirium } & \multirow[b]{2}{*}{$\mathrm{p} 1$} & \multirow[b]{2}{*}{$\mathrm{p} 2$} & \multirow[b]{2}{*}{ p3 } \\
\hline & Baseline & FU & Baseline & FU & & & \\
\hline & 42 & 42 & 14 & 14 & & & \\
\hline Red blood cell count $\left(\times 10^{6} / \mathrm{mm}^{3}\right)$ & $4.42 \pm 0.75$ & $4.21 \pm 0.72$ & $4.23 \pm 0.76$ & $4.01 \pm 0.65$ & 0.35 & 0.11 & 0.93 \\
\hline Hemoglobin (g/dl) & $12.20 \pm 2.32$ & $11.98 \pm 1.79$ & $11.49 \pm 2.42$ & $11.04 \pm 1.89$ & 0.14 & 0.32 & 0.67 \\
\hline White blood cell count $\left(\times 10^{3} / \mu \mathrm{l}\right)$ & $8.57 \pm 8.73$ & $6.35 \pm 2.20$ & $9.23 \pm 3.75$ & $8.86 \pm 3.59$ & 0.14 & 0.84 & 0.41 \\
\hline Neutrophils $\left(\times 10^{3} / \mu \mathrm{l}\right)$ & $5.47 \pm 3.63$ & $3.90 \pm 2.18$ & $7.40 \pm 3.65$ & $6.65 \pm 3.74$ & 0.006 & 0.41 & 0.46 \\
\hline Eosinophils $\left(\times 10^{3} / \mu \mathrm{l}\right)$ & $0.04 \pm 0.06$ & $0.17 \pm 0.15$ & $0.03 \pm 0.05$ & $0.13 \pm 0.12$ & 0.12 & 0.01 & 0.33 \\
\hline Lymphocytes $\left(\times 10^{3} / \mu \mathrm{l}\right)$ & $1.18 \pm 0.66$ & $1.72 \pm 0.68$ & $1.05 \pm 0.45$ & $1.45 \pm 0.72$ & 0.16 & 0.007 & 0.37 \\
\hline Monocytes $\left(\times 10^{3} / \mu \mathrm{l}\right)$ & $0.87 \pm 2.07$ & $0.49 \pm 0.19$ & $0.71 \pm 0.63$ & $0.60 \pm 0.45$ & 0.78 & 0.81 & 0.60 \\
\hline Platelet count $\left(\times 10^{3} / \mathrm{mm}^{3}\right)$ & $221.74 \pm 81.41$ & $222.46 \pm 50.84$ & $212.19 \pm 82.64$ & $221.25 \pm 100.81$ & 0.98 & 0.71 & 0.77 \\
\hline Erythrocyte sedimentation rate (mm) & $40.13 \pm 20.49$ & $39.68 \pm 20.94$ & $33.55 \pm 17.57$ & $36.80 \pm 18.42$ & 0.54 & 0.71 & 0.69 \\
\hline Reactive C protein (mg/dl) & $77.94 \pm 73.75$ & $20.11 \pm 52.59$ & $68.97 \pm 50.72$ & $66.34 \pm 61.03$ & 0.01 & 0.90 & 0.03 \\
\hline Procalcitonin (ng/ml) & $2.13 \pm 8.13$ & $0.31 \pm 1.14$ & $0.40 \pm 0.55$ & $0.39 \pm 0.60$ & 0.96 & 0.99 & 0.38 \\
\hline Prothrombin time $(\%)$ & $88.38 \pm 26.81$ & $93.30 \pm 24.90$ & $80.05 \pm 18.81$ & $80.23 \pm 17.14$ & 0.07 & 0.97 & 0.42 \\
\hline INR & $1.28 \pm 0.88$ & $1.13 \pm 0.30$ & $1.18 \pm 0.19$ & $1.18 \pm 0.20$ & 0.75 & 0.99 & 0.37 \\
\hline Activated partial thromboplastin time (s) & $38.30 \pm 22.82$ & $35.25 \pm 15.44$ & $36.11 \pm 5.78$ & $35.33 \pm 5.68$ & 0.99 & 0.80 & 0.52 \\
\hline Fibrinogen (mg/dl) & $506.73 \pm 148.30$ & $450.20 \pm 139.63$ & $432.40 \pm 166.22$ & $382.73 \pm 155.31$ & 0.16 & 0.12 & 0.86 \\
\hline D-dimer $(\mu \mathrm{g} / \mathrm{ml})$ & $2.23 \pm 5.05$ & $1.05 \pm 1.34$ & $1.73 \pm 1.47$ & $1.93 \pm 1.44$ & 0.43 & 0.87 & 0.35 \\
\hline Glycemia (mg/dl) & $104.76 \pm 23.53$ & $93.57 \pm 32.99$ & $116.44 \pm 39.60$ & $100.44 \pm 29.63$ & 0.46 & 0.12 & 0.70 \\
\hline Azotemia (mg/dl) & $55.68 \pm 43.91$ & $50.68 \pm 48.05$ & $77.25 \pm 69.76$ & $61.19 \pm 63.85$ & 0.51 & 0.11 & 0.36 \\
\hline Estimated glomerular filtration rate & $86.58 \pm 26.45$ & $97.05 \pm 20.05$ & $90.34 \pm 72.53$ & $81.23 \pm 88.19$ & 0.41 & 0.60 & 0.28 \\
\hline Creatinine (mg/dl) & $1.60 \pm 2.15$ & $1.20 \pm 1.75$ & $1.39 \pm 1.33$ & $1.34 \pm 1.46$ & 0.80 & 0.84 & 0.25 \\
\hline Albumin (g/dl) & $3.73 \pm 0.66$ & $3.54 \pm 0.82$ & $3.16 \pm 0.43$ & $3.16 \pm 0.43$ & 0.09 & 0.99 & 0.35 \\
\hline Troponin (pg/ml) & $28.85 \pm 39.78$ & $17.48 \pm 23.07$ & $23.36 \pm 19.75$ & $36.54 \pm 52.07$ & 0.19 & 0.34 & 0.12 \\
\hline Sideremia $(\mu \mathrm{g} / \mathrm{dl})$ & $38.90 \pm 22.23$ & $37.24 \pm 21.91$ & $49.07 \pm 77.05$ & $51.93 \pm 76.31$ & 0.33 & 0.37 & 0.25 \\
\hline Ferritin (ng/ml) & $679.08 \pm 1071.80$ & $532.60 \pm 870.98$ & $337.25 \pm 232.57$ & $337.25 \pm 232.57$ & 0.64 & 0.99 & 0.60 \\
\hline Neutrophil/lymphocyte ratio & $6.02 \pm 5.14$ & $2.80 \pm 2.61$ & $9.37 \pm 8.91$ & $7.12 \pm 7.67$ & 0.01 & 0.18 & 0.62 \\
\hline
\end{tabular}

For every parameter, the follow-up assessment (FU) was the last measurement available before discharge or transfer to long-term care unit. $p$ value $1=p$ value for the comparison between patients with and without delirium; $p$ value $2=p$ value for the comparison between the two times of the study; $p$ value $3=p$ value for the interaction between times of the study and presence or absence of delirium. Significant differences are highlighted in italicized character 
Studies on elderly patients suggest that inflammation plays a role in the pathophysiology of delirium [7]. This hypothesis is corroborated in our cohort by the higher levels of CRP in delirious patients as compared with patients without delirium. CRP circulating concentrations rise in response to inflammation. CRP is an acute-phase protein of hepatic origin that increases following interleukin- 6 secretion by macrophages and T cells. High serum interleukin- 6 level has been associated with increased risk of the occurrence of delirium [8]. It is suspected that systemic inflammation, which commonly characterizes critical illness, stimulates a process of deranged coagulation, whose end product is microvascular damage and thrombosis in vital organs, including the brain, contributing to the development and maintenance of delirium [9].

The use of LMWH, by preventing the development of thrombosis subsequent to the inflammatory process, may help prevent the occurrence of delirium in COVID-19 patients, with consequent possible reduction of LOS and sequelae.

Supplementary Information The online version contains supplementary material available at https://doi.org/10.1007/s10072-020-04887-4.

Authors' contributions All the authors gave substantial contributions to the conception or design of the work; or the acquisition, analysis, or interpretation of data for the work; drafting the work or revising it critically for important intellectual content; and final approval of the version to be published; agreement to be accountable for all aspects of the work in ensuring that questions related to the accuracy or integrity of any part of the work are appropriately investigated and resolved.

Data availability Data are available under request.

\section{Compliance with ethical standards}

Conflict of interest The authors declare that they have no conflict of interest.

Ethics approval The study was approved by the ethical committee of University of Brescia as the study is part of a wider study of psychiatric and neurological signs in patients with COVID-19 infection.

Consent to participate The patients gave their consent to participate to the study.
Consent for publication The patients gave their consent to have their anonymized data published.

Code availability Not applicable.

\section{References}

1. Shahid Z, Kalayanamitra R, McClafferty B, Kepko D, Ramgobin D, Patel R, Aggarwal CS, Vunnam R, Sahu N, Bhatt D, Jones K, Golamari R, Jain R (2020) COVID-19 and older adults: what we know. J Am Geriatr Soc 68:926-929. https://doi.org/10.1111/jgs. 16472

2. Simonetti AF, Viasus D, Garcia-Vidal C, Carratalà J (2014) Management of community-acquired pneumonia in older adults. Ther Adv Infect Dis 2:3-16. https://doi.org/10.1177/ 2049936113518041

3. Bellelli G, Morandi A, Di Santo SG et al (2016) "Delirium Day": a nationwide point prevalence study of delirium in older hospitalized patients using an easy standardized diagnostic tool. BMC Med 14: 106. https://doi.org/10.1186/s12916-016-0649-8

4. O'Hanlon S, Inouye SK (2020) Delirium: a missing piece in the COVID-19 pandemic puzzle. Age Ageing 49:497-498. https://doi. org/10.1093/ageing/afaa094

5. Mummery RS, Rider CC (2000) Characterization of the heparinbinding properties of IL-6. J Immunol 165:5671 LP-5675679. https://doi.org/10.4049/jimmunol.165.10.5671

6. Zhou F, Yu T, Du R et al (2020) Clinical course and risk factors for mortality of adult inpatients with COVID-19 in Wuhan, China: a retrospective cohort study. Lancet 395:1054-1062. https://doi.org/ 10.1016/S0140-6736(20)30566-3

7. McNeil JB, Hughes CG, Girard T et al (2019) Plasma biomarkers of inflammation, coagulation, and brain injury as predictors of delirium duration in older hospitalized patients. PLoS One 14:e0226412 e0226412. https://doi.org/10.1371/journal.pone.0226412

8. Simone MJ, Tan ZS (2011) The role of inflammation in the pathogenesis of delirium and dementia in older adults: a review. CNS Neurosci Ther 17:506-513. https://doi.org/10.1111/j.1755-5949. 2010.00173.x

9. Girard TD, Ware LB, Bernard GR, Pandharipande PP, Thompson JL, Shintani AK, Jackson JC, Dittus RS, Ely EW (2012) Associations of markers of inflammation and coagulation with delirium during critical illness. Intensive Care Med 38:1965-1973. https://doi.org/10.1007/s00134-012-2678-x

Publisher's note Springer Nature remains neutral with regard to jurisdictional claims in published maps and institutional affiliations. 